\title{
Dislexia en Español: estado de la cuestión
}

\author{
Francisca Serrano y Sylvia Defior
}

Departamento de Psicología Evolutiva y de la Educación.

Facultad de Psicología. Universidad de Granada

España

fdserran@ugr.es 


\section{Resumen}

La dislexia es un problema persistente en el lenguaje escrito, que consiste en una dificultad grave en el reconocimiento de palabras. Se caracteriza por un rendimiento en lectura bajo, mientras que no hay problemas en el resto de habilidades, que son normales e incluso superiores en algunos casos. Este trabajo revisa diferentes propuestas que han surgido para la definición y clarificación de las causas de la dislexia. Igualmente, se comenta la heterogeneidad de la dislexia como una característica tanto inter-población como inter-lenguas.

Palabras Clave: dislexia, sistemas ortográficos tranparentes, español, lenguaje escrito.

\section{Introducción}

El lenguaje tanto oral como escrito, constituye una habilidad humana fundamental, de ahí que el interés por su estudio haya estado presente desde los comienzos de la investigación psicológica. El uso del lenguaje oral como instrumento de comunicación nos convierte en una especie única, mientras que el desarrollo del lenguaje escrito es responsable de la evolución en el ámbito cultural de nuestra especie (Wolf, Vellutino y Gleason, 2000b). La lectura, como capacidad que permite el procesamiento del lenguaje escrito, es corresponsable de esta evolución y, por esta razón, despierta un interés paralelo al del lenguaje. Además, la indagación sobre la lectura está justificada por la redundancia, la presencia e importancia de esta capacidad en la vida cotidiana en nuestra sociedad.

El estudio de la adquisición de las habilidades lectoras tiene interés también como paso previo al análisis de las situaciones en las que se plantean dificultades en su aprendizaje, como es la dislexia. La dislexia es un problema persistente en el lenguaje escrito, caracterizado por una dificultad grave para identificar las palabras escritas que padecen algunas personas, por lo demás normales. Puede aparecer cuando la persona ya ha aprendido a leer y escribir, como consecuencia de un daño cerebral, o puede manifestarse antes de que la persona, en este caso un niño, haya aprendido a leer, causando grandes dificultades en el aprendizaje de la lectura y la escritura, cuyo origen podría radicar en deficiencias constitucionales. En el primer caso se habla de dislexia adquirida y en el segundo, de dislexia evolutiva. 
Los niños disléxicos comienzan a tener problemas en cuanto empieza la enseñanza sistemática de la lectura; su aprendizaje representa para ellos una barrera en el desarrollo académico y personal y, por tanto, preocupa a padres, a profesores y a los propios niños, conforme van creciendo y no encontrando solución a sus problemas. Su ejecución en todas las materias escolares en las que la lectura es necesaria se resiente; además, los niños comienzan a rechazar la lectura y a dedicarse a otro tipo de tareas que les reportan más satisfacciones, con lo que se produce un círculo vicioso conocido como efecto Mateo (Stanovich, 1986). Su progreso académico queda condicionado por estas dificultades y su elección profesional puede verse determinada, en buena medida, por la misma razón. La intervención para mejorar sus problemas necesita basarse en las características que presentan los niños disléxicos y en los factores causales que subyacen en la explicación de los déficit que les caracterizan.

\section{Definir la dislexia}

La concepción de este problema ha sido objeto de debate y, por ello, a lo largo de las últimas décadas, han ido surgiendo diferentes definiciones.

Una definición clásica es la que propuso la Federación Mundial de Neurología en 1968. De acuerdo con esta definición, la dislexia es un problema que se caracteriza por un déficit en el aprendizaje de la lectura a pesar de que los niños reciban una educación normal, posean una inteligencia normal y pertenezcan a un status sociocultural adecuado. La definición también apunta que estos problemas estarían causados por déficit cognitivos básicos con una base constitucional.

Esta definición está basada en el criterio de exclusión y en la discrepancia entre la habilidad lectora y la habilidad cognitiva general, es decir, el CI. En este sentido, coincide con otra de las definiciones más tenidas en cuenta en la literatura, propuesta por el Manual diagnóstico y estadístico de los trastornos mentales (DSM-IV, 1995). Según esta concepción, el trastorno de la lectura, que se sitúa dentro de los trastornos del aprendizaje, se caracteriza por un rendimiento en lectura (esto es, precisión, velocidad o comprensión de la lectura evaluadas mediante pruebas normalizadas administradas individualmente) que se sitúa sustancialmente por debajo del esperado en función de la edad cronológica, del cociente de inteligencia y de la escolaridad propia de la edad del individuo.

Algunos estudios han puesto en duda estas definiciones de la dislexia basadas en la discrepancia y en la referencia al CI como punto de corte, y han demostrado que los disléxi- 
cos tienen los mismos problemas con el lenguaje escrito sin tener en cuenta el CI (Jiménez \& Rodrigo, 1994; Siegel, 1990). Del mismo modo, estas definiciones circunscriben el déficit solo al ámbito limitado y específico del sistema cognitivo (ver Frith, 1997; Frith 1999), cuando ya está bien establecido que los déficit en dislexia se manifiestan en varios aspectos del procesamiento fonológico (Hoien \& Lundberg, 1997; Snowling, 2000).

Por esta razón, han ido surgiendo otras definiciones que dan cuenta de las características distintivas de dislexia y se alejan de la limitación de los planteamientos anteriores. Hoien \& Lundberg (1991) propusieron una definición que puede ser resumida de esta forma: "la dislexia es una dificultad en la utilización del código del lenguaje escrito, basada en una déficit en el sistema fonológico del lenguaje oral”.

Finalmente, el consenso fue obtenido con la definición propuesta por la Sociedad Orton para la dislexia (ahora Sociedad Internacional de la Dislexia) en 1994 y, más tarde, por el Instituto Nacional de la Salud. Esta definición puede ser resumida diciendo que la dislexia es un problema específico de lenguaje con una base constitucional que se caracteriza por dificultades en la descodificación de palabras simples y refleja una habilidad de procesamiento fonológico insuficiente. Dentro de esta definición cabe destacar varios aspectos importantes. En primer lugar, se centra en el nivel de reconocimiento de palabras; por otro lado, mantiene una visión modular que implica que es posible el funcionamiento inadecuado de un sistema (el de procesamiento fonológico, en este caso) mientras que permanecen intactos otros sistemas cognitivos más generales. También hay que destacar que la definición no se apoya en el CI para la determinación de los problemas de dislexia, sino precisamente en las dificultades concretas que el problema lleva asociado (descodificación, procesamiento fonológico).

Estos aspectos hacen que sea una de las definiciones más aceptadas. No obstante, la polémica se sigue manteniendo entre los autores que se ocupan de este tema (Lundberg, 1999), en cuanto a cuáles son problemas concretos más importantes que definen la dislexia o qué factores explican mejor estas dificultades.

\section{¿Qué causa la dislexia?}

La definición de dislexia no es el único punto de desacuerdo en la investigación de este tema. Otro punto de divergencia entre los autores está relacionado con los factores causales de la dislexia. Se han señalado muchas causas que podrían explicar los déficit que se dan en la dislexia, que van desde las más biológicas hasta otras más lingüísticas. 
Dentro de las causas biológicas, probablemente por influencia de las tendencias de investigación actuales, se encuentran las teorías genéticas. La investigación centrada en los estudios familiares y con gemelos (DeFries, Alarcón y Olson, 1997), sugiere el carácter hereditario de la dislexia, mientras que otras investigaciones tratan de determinar los genes que estarían a la base de este problema. Se baraja la implicación de determinados cromosomas, aunque los datos no son definitivos (Fargerheim, Tonnessen, Raeymaekers y Lubs, 1999; Olson, 1999).

Otro tipo de investigaciones de carácter biológico tratan de encontrar la causa de dislexia en el cerebro, basándose en diferencias encontradas, tanto de estructura como de función, entre los cerebros de personas disléxicas y de otras sin problemas de lectura. En este ámbito, destacan los trabajos de Galaburda, Corsiglia y Rosen (1987) que encontró diferencias en el Planum Temporale y las células magnocelulares del tálamo en análisis postmortem de cerebros disléxicos. Igualmente, los estudios de Fawcett y Nicholson (Fawcett \& Nicholson, 2001) encuentran diferencias tanto funcionales como de estructura entre los cerebelos de personas normales y disléxicos. Por último, los estudios de Stein y Walsh (1997) señalan la importancia del sistema de las células magnocelulares en la lectura, apoyándose en estudios que encuentran que cerebros de personas disléxicas tienen un menor número de estas células que los de personas normales.

Otras investigaciones han buscado explicaciones más cognitivas, a nivel de los módulos de procesamiento que se consideran claves en lectura. Estas explicaciones incluyen trabajos que abogan por un déficit del procesamiento fonológico (Lundberg \& Hoien, 2001), otros defienden como origen del problema el procesamiento visual (Pavlidis, 1981), el procesamiento rápido de estímulos (Hari \& Renvall, 2001; Wolf, 1991; Wolf, Bowers y Boddle, 2000a), el procesamiento temporal (Tallal, 1984; Farmer \& Klein, 1995, para una revisión), la capacidad para automatizar los procesos implicados en lectura (Van der Leij \& Van Daal, 1999a, 1999b), o incluso, algunos defienden la existencia de un déficit atencional de base (Hari, Valta y Uutela, 1999; Facoetti \& Turatto, 2000; Facoetti \& Molteni, 2001).

De entre todas estas explicaciones, cabe destacar, por su nivel de aceptación en la comunidad investigadora y presencia en la literatura científica, tres: la hipótesis del déficit visual, la del déficit fonológico, y la hipótesis del déficit en automatización, que se revisan brevemente a continuación. 


\section{Hipótesis del déficit visual}

Esta teoría mantiene que un déficit específico en el sistema de procesamiento visual sería la primera causa subyacente a la dislexia. Una de las primeras teorías influyentes sobre la dislexia fue la de Samuel Orton en 1925, que abogaba ya por la relación entre la dislexia y el sistema visual. Orton proponía que la causa del problema estribaría en una disfunción en la percepción y la memoria visual, que se caracterizaría por una tendencia a ver invertidas las letras y las palabras (b por d; es por se). Otras teorías más recientes, en esta misma línea han explicado los déficit disléxicos por un problema en el sistema oculomotor (Pavlidis, 1981).

Actualmente, estas ideas no se mantienen tal cual fueron formuladas, aunque sí que se considera importante la relación entre el sistema visual y la dislexia. Las nuevas formulaciones de esta hipótesis mantienen que la dislexia es causada por un déficit específico en la transferencia de la información sensorial desde los ojos hasta las áreas primarias de procesamiento visual en el cortex. Los estudios que han comparado la ejecución de niños disléxicos y normales en el procesamiento de estímulos han encontrado pruebas en este sentido (e.g. Hogben, 1997). Por ejemplo, se ha encontrado que el intervalo de procesamiento inter-estímulos en los disléxicos es más largo que en las personas normales, de forma que cuando se les presenta un segundo estímulo es posible que estén aún procesando el anterior, por lo que no podrían dedicar recursos cognitivos al nuevo estímulo.

Estas ideas están apoyadas por un sustrato neural que las investigaciones han situado en las células magnocelulares del sistema visual (Stein \& Walsh, 1997). El procesamiento cognitivo y perceptual en el cerebro al que llega la información transferida desde los ojos está formado por dos sistemas paralelos, que tienen diferentes funciones en la percepción visual: el sistema parvocelular y el magnocelular. El $1^{\mathrm{o}}$ se encarga del procesamiento visual de los colores y los detalles y el segundo, del procesamiento global del ambiente, especialmente en el caso de input sensoriales breves, como ocurre en la detección de movimientos y en la lectura.

La investigación ha mostrado que la dislexia puede estar asociada con un déficit en el sistema magnocelular. Galaburda, Rosen y Sherman (1990) encontraron claras diferencias en el sistema Magnocelular entre sujetos disléxicos y normales, mientras que no ocurrió lo mismo en el sistema Parvocelular. 


\section{Hipótesis del déficit fonológico.}

Esta hipótesis es la más aceptada en la literatura para explicar los déficit que se dan en la dislexia. De acuerdo con ella, la dislexia es causada por problemas en el sistema fonológico de procesamiento de lenguaje. Estos problemas ocasionan que el individuo tenga dificultades al usar el código alfabético para identificar las palabras (Hoien, 1999). En efecto, la comprensión y la adquisición del código alfabético requieren la habilidad para segmentar la cadena del habla en unidades del tamaño del fonema y para ponerlas en correspondencia con su representación escrita.

La dificultades fonológicas de las personas disléxicas no sólo se manifiestan en sus problemas de segmentación, sino también en la repetición de no palabras, especialmente si son complicadas y contienen combinaciones poco usuales, al leer no palabras y al escribirlas al dictado, en problemas de memoria verbal a corto plazo y en problemas atencionales y dificultades de denominación rápida (Wolf, 1991), sobre todo de colores, objetos y letras.

Los estudios neurológicos también han encontrado indicaciones a nivel cerebral que apoyan la hipótesis de un déficit fonológico. En la mayoría de las personas el hemisferio izquierdo controla las funciones de lenguaje. Dentro de él, hay algunas áreas que son más críticas que otras en la lectura. La tecnología desarrollada en los últimos años ha hecho posible la observación de imágenes cerebrales (MRI, fMRI, PET) y el registro de la actividad cerebral mientras el sujeto realiza distintos tipos de tareas (Posner \& Raichle, 1994). Diversos trabajos muestran diferencias entre sujetos disléxicos y sujetos normales en el funcionamiento cerebral de las áreas implicadas en la lectura (Posner, Abdullaev, McCandliss y Sereno, 1999; Posner \& McCandliss, 1999; Paulesu et al, 2001).

\section{Hipótesis del déficit en automatización}

A pesar de que aún existen dudas acerca del papel que juega en el desarrollo de habilidades básicas como las lectoras, se considera que la automaticidad es una característica clave en la lectura experta. De hecho, aprender a leer puede interpretarse como aprender a automatizar las habilidades de reconocimiento e identificación de palabras (Van der Leij \& Van Daal, 1999a).

En los últimos años, un sector de la investigación sobre dislexia se ha centrado en el estudio de la automatización de la lectura, con el objetivo de explorar otras dificultades que caracterizan la dislexia. Desde esta perspectiva de estudio se apunta que las personas disléxi- 
cas tienen problemas al hacer automáticos los procesos lectores. Este problema puede provocar que el reconocimiento de palabras sea más lento y torpe, lo que a su vez afectaría, finalmente, a la comprensión (e.g. Bowers \& Wolf, 1993; Wolf, 1991; Wolf \& Bowers, 1999; Wolf et al., 2000a). Se ha señalado, pues, un déficit general de automatización en la dislexia, que ha sido enfatizado en muchos trabajos con diferentes tareas (Nicolson \& Fawcett, 1990, 2001). Los disléxicos muestran problemas de automatización en el nombramiento rápido tanto con estímulos lingüísticos, como letras, como otros no lingüísticos como colores (Bowers \& Swanson, 1991), de ahí que como índice de la automatización y la fluidez lectora se utiliza la tarea de nombramiento rápido de todo tipo de estímulos. Para un revisión acerca de los problemas de automatización en dislexia puede consultarse el reciente artículo de Savage (2004).

En general, parece que la ejecución lectora de los disléxicos no alcanza el nivel de automaticidad relativamente libre de atención que parece normal en el desarrollo (Van der Leij \& Van Daal, 1999a). Por otra parte, el déficit en automatización no se limita solo al ámbito de la lectura, sino que sería ampliable a otras áreas de funcionamiento, por ejemplo a nivel motor (Wolff, Michel, Ovrut, y Drake, 1990).

El sustrato neurológico que fundamenta esta hipótesis se encuentra en los trabajos de Nicolson \& Fawcett (2001) ya citados, o los de Van der Leij \& Van Daal, (1999a) sobre el cerebelo. Según estos estudios existen diferencias a nivel cerebeloso cuando se compara la ejecución de personas disléxicas y normales en tareas motoras y de lectura.

De forma general, se observa una disparidad en las explicaciones de dislexia, que da la impresión de un cierto "caos" explicativo. Sin embargo, las diferentes propuestas explicativas sobre dislexia no tienen por qué ser contrapuestas, sino que pueden considerarse complementarias. De hecho, en las tres hipótesis comentadas se combinan explicaciones de origen biológico, de procesamiento cognitivo y comportamental que podrían aunarse en una explicación integradora.

\section{Un modelo integrador}

Frith $(1997,1999)$ propone que la explicación de la dislexia debiera hacerse desde varios niveles de descripción. La autora propone un modelo causal integrador que tiene en cuenta la influencia del ambiente e incluye los niveles de procesamiento apuntados en las tres 
hipótesis explicativas comentadas en el apartado anterior: el biológico, el cognitivo, y el conductual (ver figura 1).

Figura 1. Representación del modelo causal de dislexia de Frith (1997)

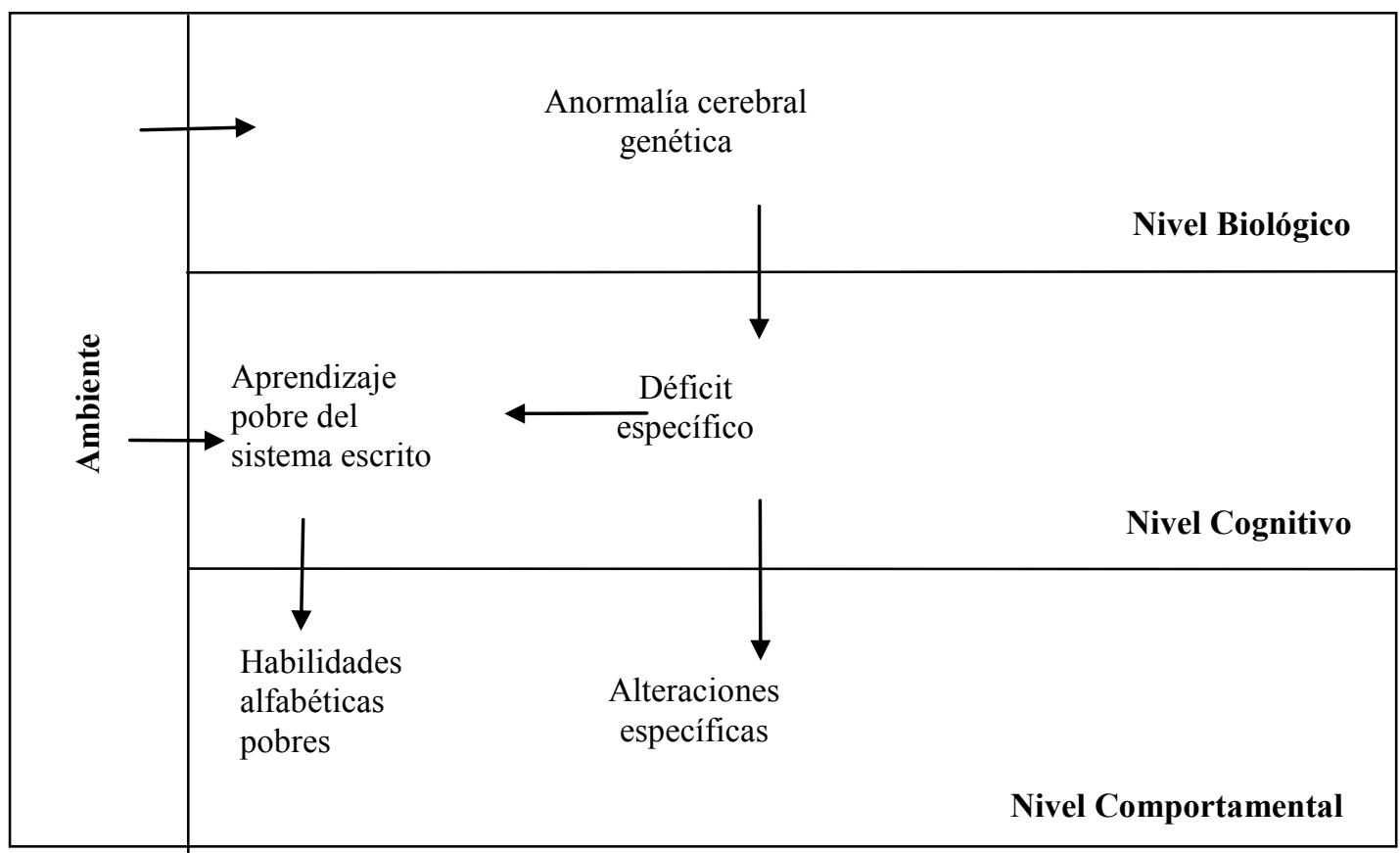

Según Frith (1997), las explicaciones genéticas y las que se basan en mecanismos y estructuras cerebrales se situarían en el nivel biológico. Las explicaciones que se basan en un déficit cognitivo de procesamiento, como la hipótesis fonológica, se situarían en el nivel cognitivo y las explicaciones que se basan en las manifestaciones consecuentes de los déficit en dislexia (rendimiento bajo en lectura, problemas de conciencia fonológica, dificultades de denominación rápida) se situarían en el nivel conductual. A su vez, estos niveles pueden estar influidos por condiciones que concurren en el ambiente, como el sistema ortográfico en el que se aprende a leer, los materiales de enseñanza de la lectura, la importancia concedida al aprendizaje de ésta, el ambiente familiar, etc.

La ventaja de este modelo es que permite integrar los distintos niveles de explicación en los que se ha hecho énfasis en la literatura científica sobre dislexia de forma separada, aportando, de esta manera, una visión integradora del problema que tiene en cuenta la diversidad de manifestaciones del mismo en la población.

\section{Heterogeneidad en dislexia}


Que exista tanta diversidad de factores en la explicación de las causas de la dislexia se ha relacionado con la propia heterogeneidad observada dentro de la población concreta de los disléxicos. Desde las primeras observaciones teóricas, los investigadores han destacado la aparente heterogeneidad de los niños con dificultades lectoras (Morris, Shaywitz, Shankweiler, Katz, Stuebing, Fletcher, Lyon, Francis y Shaywitz, 1998). Los intentos de clasificar esta variabilidad provienen de trabajos que tratan de encontrar diferentes subtipos de dislexia. Las tipologías que han surgido han utilizado diferentes criterios de clasificación. Desde los trabajos pioneros de Boder (1973) con su clasificación de los niños con dificultades lectoras en disfonéticos, deseidéticos y mixtos, se han sucedido otros trabajos que proponen una clasificación desde los referentes teóricos.

Así, Stanovich $(1988,1991)$ hipotetizó la existencia de dos tipos de dislexia a partir de la observación de que los disléxicos compartían un déficit en el dominio fonológico del lenguaje, pero variaban en otras características lingüísticas y cognitivas. Diferenció dos tipos, los que tenían problemas cognitivos específicos y restringidos a nivel fonológico, frente al resto de malos lectores ("garden-variety”) que se caracterizarían por problemas cognitivos y lingüísticos no relacionados con el nivel fonológico.

Otra clasificación fue propuesta por Wolf et al. (1994, 2000a), partiendo de la idea de la existencia de un doble déficit. Para la determinación de los subtipos de dislexia, Wolf tuvo en cuenta no solo la evaluación de las habilidades fonológicas, sino también de las habilidades de denominación rápida. A partir de estas dos variables encontró tres tipos de sujetos con dificultades lectoras: los que solamente tenían problemas en las habilidades fonológicas, los que únicamente tenían problemas en las habilidades de nombramiento rápido y los que tenían problemas en ambas habilidades.

Una clasificación clásica de los disléxicos es la que propusieron Castles \& Coltheart (1993). Estos autores desarrollaron su tipología a partir de las investigaciones realizadas en los problemas de lenguaje consecuentes a un daño cerebral y teniendo como referencia el marco explicativo del modelo de doble ruta.

Los estudios con personas con dislexia adquirida habían encontrado dos patrones de síntomas que cumplían una doble disociación (las referencias de estos trabajos pueden encontrarse en Castles \& Coltheart, 1993). En primer lugar, había informes de personas que podían leer en voz alta palabras regulares y no palabras, pero que tenían problemas con las palabras irregulares. Los errores más comunes de estas personas eran la regularización de las palabras 
irregulares, es decir, las pronunciaban de acuerdo con las reglas de conversión G-F, sin atender a su carácter especial. Este patrón de síntomas fue llamado dislexia superficial y es el que surge debido a un daño en las zonas cerebrales implicadas en el procedimiento léxico de lectura. Por otro lado, otros autores han descrito casos de personas que podían leer sin problema tanto palabras regulares como irregulares, pero que tenían dificultad con las no palabras, dificultades que seguían a un daño en las áreas cerebrales implicadas en el procedimiento subléxico de lectura. A este tipo de dislexia se le llamó fonológica.

Se ha encontrado que esta doble disociación también se cumple cuando los problemas lectores no son consecuencia de un daño cerebral, es decir, cuando hablamos de dislexias evolutivas. A pesar de la controversia que sigue existiendo sobre el tema, esta idea está muy afianzada en la literatura (Calvo, 1999; Castles \& Coltheart, 1993; Genard, Mousty, Content, Alegria, Leybaert y Morais, 1998; Manis, Seidenberg, Doi, McBride-Chang y Petersen, 1996; Manis, Seidenberg, Stallings, Joanisse, Freedman, Curtin y Keating, 1999; Morris et al., 1998; Stanovich, Siegel y Gotardo, 1997).

En su estudio clásico, Castles \& Coltheart (1993) examinaron un total de 112 participantes, 56 de los cuales eran disléxicos y tenían un retraso lector importante, utilizando una batería de pruebas que les permitía una evaluación separada del funcionamiento de las dos vías de lectura propuestas en la literatura científica. Dentro de esta batería incluían pruebas de lectura de no palabras pronunciables (para la evaluación de la ruta subléxica) y de lectura de palabras irregulares (para la evaluación de la ruta léxica). Los resultados que encontraron apoyan la existencia de los dos subtipos de dislexia encontrados en los pacientes de daño cerebral.

Los trabajos posteriores de Manis et al. (1996; 1999), han validado esta división partiendo de la perspectiva de los modelos conexionistas, en lugar de los de doble ruta. De esta manera, la clasificación más utilizada de los problemas disléxicos distingue entre disléxicos fonológicos y superficiales. Los disléxicos fonológicos solo pueden leer por la ruta léxica, ya que está alterada la fonológica, y se caracterizan porque leen bien palabras familiares pero no pueden leer pseudopalabras, ni palabras desconocidas, debido a que no pueden utilizar el mecanismo de conversión de grafema-fonema. Son sensibles al efecto de frecuencia, pero no al de longitud de las palabras ni de regularidad. Cometen errores visuales en las pseudopalabras que se parecen a palabras, con abundantes lexicalizaciones (antiguo por artiguo; playa por blaya) y en la lectura de palabras parecidas (firme, por forma). También comenten errores 
morfológicos o derivativos: mantienen la raíz pero cambian el sufijo (andaba, por andar; salíamos por salido) y tienen más errores en palabras función que en las de contenido. Los disléxicos superficiales pueden leer por el procedimiento fonológico pero no por el léxico, y por ello, normalmente son incapaces de reconocer una palabra como un todo. Estas personas leen mejor las palabras regulares, sean familiares o no, pueden leer pseudopalabras y sus errores más frecuentes son de omisión, adición o sustitución de letras. Además se caracterizan por la regularización de las palabras irregulares y la confusión de homófonos, porque el acceso léxico está guiado por el sonido y no por la ortografía de la palabra. También es posible encontrar un grupo de disléxicos mixto, en el que se den déficit de los dos tipos.

\section{Dislexia ¿déficit o retraso?}

La distinción entre los subtipos de dislexia está relacionada con otro debate teórico, el que se establece entre los que la consideran que existe un déficit específico en el lenguaje escrito y los que consideran que la dislexia es un mero retraso madurativo que podría ser remediado con el paso del tiempo y una intervención específica en estos problemas (Bandian, 1996; Gottardo, Chiappe, Siegel y Stanovich, 1999; Jacobson, 1999; Jiménez \& Hernández, 2001; Metsala, Stanovich y Brown, 1998; Samuelsson, Finnstroem, Leijon y Mard, 2000; Treesoldi, Stella y Faggela, 2001). La idea de que la dislexia es un problema de retraso en el desarrollo se apoya en la observación de los niños que son diagnosticados con dislexia superficial (Samuelsson et al, 2000). Sus problemas no son tan llamativos, en lo que se refiere a precisión lectora, como los de niños con dislexia fonológica u otros problemas lectores, y se ha encontrado que, con el tiempo y trabajo, llegan a alcanzar el nivel lector de sus compañeros de la misma edad. Sin embargo, parece como si los estudios que mantienen esta hipótesis no tuvieran en cuenta los problemas de estos niños en velocidad y fluidez lectora. Tampoco existen muchas pruebas de lectura que midan la velocidad lectora, además de la precisión, ni que sean aplicables a personas más allá de la escolaridad obligatoria. Del mismo modo, esta hipótesis se mantiene desde estudios que se han realizado en sistemas ortográficos trasparentes (Jiménez \& Hernández, 2001; Treesoldi et al., 2001; Serrano \& Defior, enviado; Wimmer, 1993), en los que se observan características de dislexia menos graves que las que presentan niños de ortografías más opacas, como el inglés, y que mejoran con el tiempo y el tratamiento. La hipótesis de que la dislexia se debe a un déficit respecto al patrón de desarrollo normal y no a un mero retraso, viene apoyada por los estudios con personas disléxicas mayores, en los que siguen persistiendo los problemas a pesar de haber recibido una formación académica normal y no presentar otros problemas fuera del ámbito de la lectoescritura (Jackson \& Do- 
llinger, 2002; Kitzen, 2001; Wilson \& Lesauz, 2001). Aunque la polémica no se ha solucionado, los estudios con disléxicos adultos y con niños encuentran que algunos déficit en dislexia son tan graves que no pueden ser explicados por un retraso, sino por una desviación del patrón normal de desarrollo, por lo que dan apoyo a la hipótesis del déficit específico (Bandian, 1996; Gottardo et al., 1999; Jacobson, 1999;. Metsala et al. 1998).

La clarificación de esta discusión es importante de cara a la planificación del tratamiento para la dislexia. Éste oscilaría entre el mero apoyo, que ayude a paliar el retraso, y las acciones específicas que actuaran sobre el déficit, en cada caso. En este sentido, es necesario seguir investigando con estudios que utilizan un diseño de edad lectora, así como con trabajos en sistemas escritos transparentes.

\section{La dislexia en diferentes sistemas escritos.}

La mayoría de los estudios realizados en el ámbito del aprendizaje de la lectura y en el de las dificultades lectoras se han llevado a cabo con personas de habla inglesa. Sin embargo, estudios translingüísicos (Müller \& Brady, 2001; Öney \& Durgunuglu, 1997; Seymour, Aro y Erskine, 2003), han sugerido diferencias entre los sistemas escritos, de forma que, se puede pensar que los resultados de los estudios de lengua inglesa no son totalmente aplicables a los de otras ortografías. Según este tipo de estudios, el desarrollo de las habilidades lectoras se produce de forma diferente en las distintas ortografías, estando influido por el sistema ortográfico y el ambiente lingüístico dentro del cual se desarrolla el lector (Müller \& Brady, 2001).

Los sistemas ortográficos se distribuyen en un continuo de opacidad-transparencia de acuerdo con el grado de respeto al principio alfabético. De esta forma, las ortografías transparentes son aquellas en las que las correspondencias grafema-fonema son uno a uno. Las ortografías opacas serían aquellas en las que a un mismo fonema le corresponden varios grafemas y a un grafema le pueden corresponder varios fonemas. Los distintos sistemas escritos se distribuyen en este continuo, acercándose o alejándose de sus extremos en función de sus características. Existe bastante consenso en la posición de cada uno en este rango de transparenciaopacidad. Por lo que respecta a las ortografías europeas, las que estarían más cerca del extremo transparente serían el finés, el griego, el italiano y el español; las que se acercarían más al extremo opaco serían el inglés, el francés, el danés y el portugués (Seymour et al., 2003). 
La importancia de la realización de estos trabajos que comparan diferentes ortografías estaría justificada por el hecho de que las características particulares de cada sistema escrito podrían afectar al modo en el que se desarrolla la lectura en ellos, como ya se ha comentado. La transparencia-opacidad de las ortografías podría influir en los correlatos tempranos de la lectura. Mientras que las habilidades de descodificación y los factores fonológicos serían importantes indicadores de la lectura en la ortografía inglesa (Adams, 1990; Bradley \& Bryant, 1983), en las ortografías más trasparentes, en las que la descodificación resulta más fácil que en las opacas, esta habilidad podría tener menos influencia. Igualmente, el carácter trasparente-opaco de las lenguas puede influir en el desarrollo de la lectura desde sus primeros estadios hasta la lectura fluida. Como en una ortografía transparente es más fácil alcanzar el grado de lectura experta que en una opaca, la consecuencia sería que los lectores principiantes dispondrán de más recursos cognitivos libres para dedicarlos al procesamiento de habilidades de alto nivel como la integración de textos y la comprensión (Stanovich, 1993)

La dislexia también podría verse influida por las diferencias entre los sistemas ortográficos. Se ha estimado que la prevalencia de las dificultades de aprendizaje en los distintos países refleja las diferencias en la complejidad ortográfica de éstos; la dislexia es más común en países donde la ortografía es compleja, es decir, en los que tienen un sistema escrito más opaco, que en aquellos en los que es más transparente. Incluso se han señalado correlatos causales de la dislexia más importantes que otros en función del sistema escrito que se esté considerando. En las ortografías opacas, el criterio de comparación del logro lector en niños con dislexia y niños normales es la precisión en la ejecución lectora. En las ortografías trasparentes, la precisión lectora es un factor menos importante, siendo más determinante la baja velocidad lectora. Los trabajos en este tipo de sistemas escritos, como el alemán (Wimmer, 1993; Wimmer \& Mayringer, 2001), el finés (Holopainen, Ahoen y Lyytinen, 2001; Müller \& Brady, 2001), el italiano (Treesoldi et al., 2001) y el español (Jiménez y Hernández, 2001; Serrano y Defior, enviado; en prensa) apoyan estas ideas.

En cualquier caso, y a pesar de estas diferencias, se sigue manteniendo que las dificultades lectoras en dislexia tienen su base principal en un déficit de procesamiento fonológico, independientemente del sistema ortográfico. Un estudio reciente de Paulessu et al. (2001) mostró que, aunque las manifestaciones de dislexia podían cambiar en función del sistema ortográfico que se estuviera considerando, el déficit cognitivo principal y las bases cerebrales de este problema eran universales. En este estudio, participaron niños disléxicos y normales de países con ortografías transparentes (italianos) y opacas (ingleses y franceses). Los disléxi- 
cos italianos tuvieron mejor ejecución en lectura (mediada solo por el número de respuestas correctas) que los ingleses y franceses, pero cuando los tres tipos de disléxicos eran comparados con los niños normales de sus respectivos países, el patrón de resultados de las tres nacionalidades (diferencias entre disléxicos y normales) era semejante.

Las diferencias entre los sistemas escritos también pueden influir en la determinación de los subtipos de dislexia. Según Wimmer (1993) un disléxico fonológico alemán tendrá características diferentes que un disléxico fonológico inglés, en el sentido de que sus dificultades en el procesamiento fonológico serán mayores, ya que han persistido a pesar de la trasparencia del idioma. Por otro lado, si la habilidad en el manejo de las reglas de correspondencia grafema-fonema es la característica que distingue a los dos subtipos de disléxicos, el carácter transparente del idioma también podría influir en el cambio de las diferencias entre los dos subtipos. Por último, las diferencias entre los sistemas escritos también podrían influir en la medida utilizada para la comparación de los problemas disléxicos. Mientras que en las ortografías más opacas la medida más utilizada son los errores en la lectura, en las más transparentes sería más apropiado tener en cuenta el tiempo de lectura (Treesoldi y cols. 2001).

El español es un sistema escrito que se sitúa en el continuo comentado anteriormente en la posición cercana al extremo de trasparencia. Algunos estudios en español (Calvo, 1999; Jiménez \& Hernández, 2001) han prestado apoyo empírico a las conclusiones comentadas en las ortografías trasparentes. En un estudio reciente, Serrano \& Defior (en prensa) examinaron las habilidades de conciencia fonológica de niños disléxicos en un diseño de edad lectora equivalente. Los resultados mostraron que los problemas de los niños disléxicos fueron más importantes cuando se analizaba la medida de velocidad de la tarea fonológica, en consonancia con otros estudios en alemán y en italiano. Otro estudio con el mismo diseño (Serrano \& Defior, enviado) encontró dificultades en la medida de precisión en una tarea de lectura de no-palabras, probablemente debidas a las altas demandas fonológicas de la tarea, ya que los ítems estaban formados sin seguir las reglas fonotácticas del construcción de palabras en español. Aun así, en esta misma tarea, y en otras comentadas en el mismo trabajo, las dificultades de los disléxicos fueron más llamativas en las medidas de velocidad.

\section{Conclusión}

La dislexia evolutiva es un problema grave en el ámbito académico, ya que afecta a una habilidad que es tanto herramienta como objetivo en el proceso de enseñanza-aprendizaje. Como tal, preocupa tanto en la comunidad escolar como en la vida familiar. A pesar de ello, 
su concepción y la determinación de sus causas son aun temas polémicos que impulsan la investigación y la discusión científica. Por otra parte, recientes estudios translingüísticos han suscitado la necesidad de tener en cuenta las características del sistema escrito a la hora de definir las características de la dislexia.

El español es un sistema ortográfico considerado trasparente. El aprendizaje del código alfabético está apoyado por la casi completa correspondencia unívoca entre los grafemas y los fonemas y es considerado más fácil y rápido que en otras ortografías más opacas. No obstante, también existen casos de problemas de aprendizaje de la lectoescritura. En la dislexia evolutiva en español existe un déficit de procesamiento en el ámbito fonológico que se caracteriza fundamentalmente por problemas de velocidad. Actualmente, la velocidad no se tiene muy en cuenta en la evaluación y diagnóstico de la competencia lectora y esto tiene como consecuencia que, aparentemente, algunos niños tienen pocos problemas comparados con algunos de sus compañeros, lo que dificulta su identificación como disléxicos y el planteamiento de una intervención que les beneficie.

En este sentido, debería tenerse en cuenta que en los sistemas ortográficos trasparentes, los problemas de velocidad en la lectura son más esclarecedores que los de precisión. Igualmente, desde el ámbito educativo, sería importante tener en cuenta estos problemas, tanto en la evaluación de las dificultades y de los logros escolares de los niños, como en la prestación de apoyo que puede hacerse desde la escuela y la familia. Respecto a la intervención, habría que potenciar las habilidades de automatización en los niños disléxicos, a la vez que no saturar su capacidad de procesamiento con tareas irrelevantes que les ocupan recursos necesarios para el resto de tareas.

\section{Referencias bibliográficas}

Adams, M. (1990). Beginning to read: Thinking and learning about print. Cambridge: MIT Press.

American Pyachiatrich Association (1995) DSM-IV. Manual diagnóstico y estadístico de los trastornos mentales. Barcelona, Masson.

Badian, N. (1996) Dislexia: A variation of the concept at two age levels. Journal of Learning disabilities, 29(1), 102-112.

Boder, E. (1973). Developmental dyslexia: a diagnostic approach based on three atypical reading-spelling patterns. Developmental Medicine and Child Neurology, 15, 663687. 
Bowers, P. G. \& Swanson, L. B. (1991). Naming speed deficits in reading disability: Multiple measures of a singular process. Journal of Experimental Child Psychology, 51, 195219.

Bowers, P.G. \& Wolf, M. (1993). Theoretical links among naming speed, precise timing mechanisms and orthographic skill in dyslexia. Reading and Writing: An Interdisciplinary Journal, 5(1), 69-85.

Bradley, L. \& Bryant, P. E. (1983). Categorising sounds and learning to read: a causal connexion. Nature, 301, 419-421.

Calvo Rodríguez, A. (1999). Adquisición de la le lengua castellana: Perfiles cognitivos de aprendices con dificultades. Tesis Doctoral, Universidad de Murcia.

Castles, A., \& Colheart, M. (1993). Varieties of develomental dyslexia. Cognition, 47, 149180.

De Fries, J., Alarcon, M. y Olson, R.K. (1997). Genetic aetiologies of reading and spelling deficits: Developmental differences. En C. Hulme \& M. Snowling (Eds.), Dyslexia. Biology, cognition and intervention (pp. 20-37). London: Whurr Publishers.

Faccoeti, A. \& Molteni, M. (2001). The gradient of visual attention in develomental dyslexia. Neuropsychologia, 39, 352-357.

Facoetti, A. \& Turatto, M. (2000). Asymetrical visual fields distribution of attention in dyslexic children: a neuropsychological study. Neuroscience Letters, 290, 216-218.

Fargerheim, T., Tonnessen, F.E., Raeymaerkers, P. y Lubs, H. (1999). Exclusion of linkage to $1 \mathrm{p}, 6 \mathrm{p}$ and chromosome 15 in a large norwegian family with dyslexia. En I. Lundberg, F.E. Tonnesen y I. Austad (Eds.), Dyslexia: Advances in theory and practice. London: Kluwer Academic Publishers.

Farmer, M. E. \& Klein, R. M. (1995). The evidence for a temporal processing deficit linked to dyslexia: A review. Psychonomic Bulletin \& Review, 2(4), 460-493.

Fawcett, A. J. \& Nicolson, R. I. (2001). Dyslexia:The role of the cerebellum. En A. Fawcett (Ed.), Dyslexia. Theory and Good Practice. .London: Whurr Publishers.

Frith, U. (1997). Brain, mind and behaviour in dyslexia. En C. Hulme \& M. Snowling (Eds.), Dyslexia: Biology, cognition and intervention (pp. 1-19). London: British Dyslexia Association.

Frith, U. (1999). Paradoxes in the definiton of dyslexia. Dyslexia, 5, 192-214.

Galaburda, A. Corsiglia, J. y Rosen, G. (1987). Planum Temporale assymetry, reapprasial since Geschwind and Levitsky. Neuropsychologia, 25, 853-868. 
Galaburda, A., Rosen, G. y Sherman, A. (1990). Individual variability in cortical organizations: Its relationship to brain laterality and implications to function. Neuropsychologia, 28, 529-546.

Genard, N., Mousty, P., Content, A., Alegria, J., Leybaert, J., y Morais, J. (1998). Methods to establish subtypes of developmental dyslexia. In P. Reitsma \& L. Verhoeven (Eds.), Problems and interventions in literacy development (pp. 163-176). Netherlands: Kluwer Academic Publishers.

Gottardo, A., Chiappe, P, Siegel, L.S., Stanovich, K.E. (1999). Pattern of word and nonword processing in skilled and less-skilled readers. Reading and Writing: An interdisciplinary journal, 11(5-6), 465-487.

Hari, R. \& Renvall, H. (2001). Impaired processing of rapid stimulus sequences in dyslexia. Trends in Cognitive Sciences, 5(12), 525-532.

Hari, R., Valta, M., y Uutela, K. (1999). Prolonged attentional dwell time in dyslexic adults. Neuroscience letters, 271, 202-204.

Hogben, J. (1997). How does a visual transient deficit affects reading? En C. Hulme \& M. Snowling (Eds.), Dyslexia. Biology, cognition and intervention (pp. 20-37) London: Whurr Publishers.

Hoien, T. \& Lundberg, I. (1991). Dysleksi. Oslo: Gyldendal.

Hoien, T. \& Lundberg, I. (1997). Dysleksi. Fra teori til praksis. Oslo: Ad Notam, Gyldendal.

Hoien, T. (1999). Theories of deficits in dyslexia. En I. Lundberg, F.E. Tonnesen y I. Austad (eds.) Dyslexia: Advances in theory and practice. London: Kluwer Academic Publishers.

Holopainen, L., Ahonen, T., y Lyytinen, H. (2001). Predicting delay in reading achievement in a highly transparent language. Journal of Learning Disabilities, 34(5), 401-413.

Jackson, N.E. \& Doellinger, H. L. (2002). Resalient readers? University who are poor recoders but sometimes good text comprehenders. Journal of Educational Psychology, 94(1): 64-78.

Jacobson, C. (1999). How persistent is reading disability? Individual growth curves in reading. Dyslexia, 5, 78-93.

Jiménez, J. E. \& Rodrigo, M. (1994). Is it true that the differences in reading performance between students with and without LD cannot be explained by IQ?. Journal of Learning Disabilities, 27, 155-163.

Jiménez González, J. E. \& Hernández Valle, I. (2000). Word Identification and Reading disorders in the Spanish Language. Journal of Learning disabilities, 33(1), 44-60. 
Kitzen, K.R. (2001). Prosodic sensitivity, morphological ability, and reading ability in young adults with and without chilhodd histories of reading difficulty. Dissertation $A b$ stract International Section A: Human and Social Sciences, 62 (2-A): 460

Lundberg, I. (1999). Definitions of dyslexia. En I. Lundberg, F.E. Tonnesen y I. Austad (Eds.) Dyslexia: Advances in theory and practice. London: Kluwer Academic Publishers.

Lundberg, I. \& Hoien, T. (2001). Dyslexia and phonology. En A. Fawcett (Ed.), Dyslexia. Theory and Good Practice. . London: Whurr Publishers.

Manis, F. R., Seidenberg, M. S., Doi, L.M., McBride-Chang, C. y Petersen, A. (1996) On the bases of two subtypes of development dyslexia. Cognition, 58, 157-195.

Manis, F. R., Seidenberg, M. S., Stallings, L., Joanisse, M., Freedman, L., Curtin, S. y Keating, P. (1999). Development of dyslexic subgroups: a one-year follow up. Annals of Dyslexia, 49, 105-134.

Metsala, J.L., Stanovich, K.E., Brown, G.D.A. (1998). Regularity effects and the phonological deficit model of rg disabilities: A metaanalitic review. Journal of Educational Psychology, 90(2), 279-293

Morris, R. D., Shaywitz, S. E., Shankweiler, D. P., Katz, L., Stuebing, K. K., Fletcher, J. M., Lyon, G. R., Francis, D. J., y Shaywitz, B. A. (1998). Subtypes of reading disability: variability around a phonological core. Journal of Educational Psychology., 90(3), 347-373.

Müller, K., \& Brady, S. (2001). Correlates of early reading performance in a transparent orthography. Reading and Writing: An Interdisciplinary Journa., 14, 757-799.

Nicholson, R.I. \& Fawcett, A.J. (1990). Automaticity: A new framework for dyslexia research. Cognition, 35, 159-182.

Nicholson, R.I. \& Fawcett, A.J. (2001). Dyslexia as a learning disability. En A.J. Fawcett (Ed.), Dyslexia: Theory and good practice (pp. 141-159). London: Whurr.

Olson, R.J. (1999). Research on reading disabilities in the Colorado learning disabilities reseach center. En I. Lundberg, F.E. Tonnesen y I. Austad (Eds.) Dyslexia: Advances in theory and practice. London: Kluwer Academic Publishers.

Öney, B. \& Durgunoglu, A. (1997). Beginning to read in Turkish: A phonologically transparent orthography. Applied Psycholinguistics, 18(1), 1.

Paulessu, E., Démonet, J.-F., Fazio, F., McCrory, E., Chanoine, V., Brunswick, N., Cappa, F., Cossu, G., Habib, M., Frith, C. D. y Frith, U. (2001). Dyslexia: Cultural Diversity and Biological Unity. Science, 291, 2165-2167. 
Pavlidis, G.Th. (1981). Do eye movements hold the key of dyslexia? Neuropsychologia, 19, $57-64$.

Posner, M. \& Raichle, M. (1994) Images of Mind. Madrid: Ariel.

Posner, M., Abdullaev, Y.G., McCandliss, B.D. y Sereno, S.C. (1999). Anatomy, circuitry and plasticity of word reading. En J. Everatt. (Ed), Reading and Dyslexia: Visual and attentional processes. London and New York: Routledge.

Posner, M. \& McCandliss, B.D. (1999). Brain circuitry during reading. En Klein, R. \& McMullen, P. (Eds), Converging methods for understanding reading and dislexia. (pp. 305-337). Cambridge: MIT Press.

Samuelsson, S., Finnstroem, O., Leijon, I. y Mard, S. (2000) Phonological and surface profiles of reading disabilities among very low birth weight children: Converging evidence for the developmental lag hypothesis. Scientific Studies of Reading, 4(3), 197217.

Savage, R. (2004). Motor skills, automaticity and developmental dyslexia: A review of the research literature. Reading and Writing: An Interdisciplinary Journal, 17, 301-324

Serrano, F \& Defior, S. (en prensa). Las habilidades de conciencia fonológica en niños disléxicos españoles: ¿déficit o retraso?. XXII Congreso Internacional de la Asociación Española de Lingüística Aplicada (AESLA) "Perspectiva interdisciplinar y tecnologías emergentes". Universidad de Valencia.

Serrano, F \& Defior, S. (enviado). Dyslexia Speed Problems in a Transparent Orthography. Journal of Educational Psychology.

Seymour, P. H. K. (1986). Cognitive Analysis of Dyslexia. London and New York: Routledge \& Kegan Paul.

Seymour, P.H.K., Aro, M. y Erskine, J.M. (2003). Foundation literacy acquisition in European orthographies. British Journal of Psycholog, 94, 143-174.

Siegel, L. (1990). IQ and learning disabilities: R.I.P. In: H. Swanson \& B. Keogh (eds.), Learning disabilities: theoretical and research issues (pp. 111-128). Hillsdale, NJ: Erlbaum.

Snowling, M.J. (2000). Dyslexia. Oxford: Blackwell.

Stanovich, K. E. (1986). Matthew effects in reading: Some consequences of individual differences in the acquisition of literacy. Reading Research Quarterly, 19, 278-301.

Stanovich, K.E. (1988). Explaining the differences between dislexic and the garden-variety poor readers: The phonological core variable difference model. Journal of learning disabilities, 21, 590-604. 
Stanovich, K.E. (1991). Discrepancy definitions of reading disability: Has intelligence led us astray? Reading Research Quarterly, 26, 1-29.

Stanovich, K.E. (1993). Romance and reality, The reading Teacher, 47, 278-291.

Stanovich, K. E., Siegel, L. S., y Gottardo, A. (1997). Converging evidence for phonological and surface subtypes of reading disability. Journal of Educational Psychology, 89(1), 114-127.

Stein, J. \& Walsh, V. (1997). To see but not to read; the magnocellular theory of dyslexia. Trends in cognitive neurosciences, 20(4), 147-152.

Tallal, P. (1984). Temporal or phonetic processing deficit in dyslexia? That is the question. Applied Psycholinguistics, 5, 182-198.

Tressoldi, P., E., Stella, G., y Faggella, M. (2001). The development of reading speed in Italians with Dyslexia: A longitudinal study. Journal of learning disabilities, 34(5), 414-417.

Van der Leij, A., \& Van Daal, H. P. (1999a). Automaticity, automatization and Dyslexia. En I. L. Kundberg, F. E. Tonnessen, y I. Austad (Eds.), Dyslexia: Advances in Theory and Practice. Dordrecht: Kluwer academic publishers.

Van der Leij, A., \& Van Daal, H. P. (1999b). Automatization aspect of Dyslexia: Speed limitations in Word identification, sensitivity to increasing task demands, and orthographic compensation. Journal of Learning Disabilities, 32, 417-428.

Wilson, A. M. \& Lesaux, N.K. (2001). Persisitence of phonological processing defficits in college student with dyslexia who have age-appropiate reading skills. Journal of Learning Disabilities, 34(5), 394-400.

Wimmer, H. (1993). Characteristics of developmental dyslexia in a regular writing system. Applied Psycholinguistics, 14, 1-33.

Wimmer, H. \& Mayringer, H. (2001). Is the Reading-Rate problem of German Dyslexic Children Caused by Slow Visual Processes? En M. Wolf (Ed.), Dyslexia, Fluency and the Brain. . N.Y.: York Press.

Wolf, M. (1991). Naming speed and reading: The contribution of the cognitive neurosciences. Reading Research Quarterly, 26(2), 123-141.

Wolf, M., Feil, C., Lotz, R. y Boddle, K. (1994) Towards a more universal understanding of the developmental dyslexias: The contributions of orthographics factors. En V.W. Berninger (Ed.), The varieties of orthographic knowledge (vol 1, pp.137-171) Dordrecht, The Netherlands: Kluwer Academic. 
Wolf, M. \& Bowers, P. (1999). The double deficit hypothesis for the developmental dyslexias. Journal of Educational Psychology, 91, 1-24.

Wolf, M., Bowers, P. G., y Boddle, K. (2000a). Naming-Speed Processes, Timing, and Reading: A conceptual review. Journal of Learning disabilities, 33(4), 387-407.

Wolf, M., Vellutino y Gleason, J. B. (2000b). Una explicación psicolingüística de la lectura. In J. B. Gleason \& N. Bernstein (Eds.), Psicolingúística (pp. 433-468). Madrid: McGraw-Hill

Wolff, P.H., Michel, G.F., Ovrut, G.F. y Drake, C. (1990). Rate and timing of motor coordination in developmental dyslexia. Developmental Psychology, 26(3), 349-359. 